\title{
Infectious mononucleosis-like syndrome with high lymphocytosis and positive IgM EBV and CMV antibodies in a three-year-old girl
}

\author{
JOANNA ALICJA KRUPKA ${ }^{1}$, KATARZYNA SAMELSKA ${ }^{2}$, ANNA TOMASIK', \\ ANNA STELMASZCZYK-EMMEL ${ }^{3}$, KATARZYNA PAWELEC ${ }^{2}$
}

${ }^{1}$ Student's Scientific Group ‘SPHEROCYTE’ Department of Paediatric Haematology and Oncology, Warsaw Medical University, Poland ${ }^{2}$ Department of Paediatric Haematology and Oncology, Warsaw, Medical University, Poland

${ }^{3}$ Department of Laboratory Diagnostics and Clinical Immunology of Developmental Age, Warsaw Medical University, Poland

\begin{abstract}
Primary Epstein-Barr virus (EBV) and cytomegalovirus (CMV) infection usually affects preadolescent children or young adults, causing similar clinical presentation. Signs and symptoms are typically mild, and the majority of clinical and laboratory findings resolve spontaneously within one month after onset. In adulthood, the risk of fulminant EBV infection and severe complications is much higher, which may be related to increasing memory $C D 8+T$-cell population with age. It is still not clear what exactly triggers $T$-cell clonal proliferation. Animals model studies on heterologous immunity between viruses revealed that pre-existing memory $T$-cells may contribute to excessive immune response during subsequent infection with a new, unrelated pathogen.

A 3.5-year-old girl was admitted to hospital with a suspicion of lymphoproliferative disorder. Peripheral blood smear revealed a massive lymphocytosis $(61,600 \mathrm{cells} / \mu \mathrm{l})$ with $62 \%$ share of atypical lymphocytes. The clinical presentation and positive EBV and CMV IgM test strongly suggested infectious mononucleosis syndrome as a result of EBV and CMV coinfection.
\end{abstract}

Key words: infectious mononucleosis, EBV, CMV.

(Cent Eur J Immunol 2017; 42 (2): 210-212)

\section{Introduction}

Infectious mononucleosis (IM) is an acute infectious disease predominantly caused by the Epstein-Barr virus (EBV). The virus typically spreads through direct contact with infected saliva or blood, but sometimes transmission can occur via shared food and contaminated objects of everyday use such as toothbrushes or kitchenware. Even one year after primary infection high levels of EBV can be detected in saliva. The incubation period from the time of exposure to the first noticeable symptoms is relatively long, as it usually takes between 30 and 50 days. Therefore, the source of an infection remains undetermined in most cases [1].

Primary EBV infection usually affects preadolescent children and young adults; however, the clinical presentation among different age groups is significantly diverse. Childhood infection is typically subclinical, manifested by mild, flu-like illness, while adolescents and adults often present with pharyngitis, cervical lymph node enlargement, splenomegaly, headache, fatigue, and fever. Com- plications including jaundice, splenic rupture, periorbital oedema, neurological symptoms, haemolytic anaemia, or coagulation disturbances are rarely observed. Previous research revealed that immune response against infected B-cells, predominantly mediated by NK cells, CD4+, and CD8+ T-cells, can be a double-edged sword. The cellular immunity is a major factor that prevents EBV from spreading, but it may also contribute to apparent symptoms of infection due to excessive release of cytokines: interleukin-1 (IL-1), interleukin-6 (IL-6), and tumour necrosis factor (TNF). Atypical lymphocytosis, mainly composed of activated CD8+ T-cells, is not pathognomonic for EBV. Primary HIV infection, cytomegalovirus (CMV), viral hepatitis, mumps, rubella, toxoplasmosis, and drug reaction should be taken into consideration during differential diagnosis [2, 3].

IM is primarily diagnosed by symptoms evaluation; however, IM syndrome can be rarely attributable to other common contagious agents such as CMV, HHV-6, Toxoplasma gondii, HIV, or adenovirus. Therefore, the cause

Correspondence: Katarzyna Pawelec, Department of Paediatric Haematology and Oncology, Warsaw Medical University,

Żwirki i Wigury 63 A, 02-091 Warsaw, Poland, e-mail: katarzyna.pawelec@litewska.edu.pl

Submitted: 6.03.2017, Accepted: 11.04.2017 
of infection cannot be determined without specific assays. Immunoassays for detecting VCA IgM, VCA IgG, and EBNA-1 IgG consist of a routinely performed serological test to confirm clinical suspicion. For diagnosis in atypical cases, PCR examination may be determinative. Heterophile antibody tests are not a reliable diagnostic test for children below four years old because of the high risk of false negative results [2].

In general, IM is a self-limiting syndrome, so management can be primarily supportive. Clinical benefits of acyclovir, valacyclovir, or nucleoside analogues as a causative treatment have not yet been proven, so antiviral agents are not routinely recommended during the acute infection. The use of corticosteroids is reserved for IM-related airways obstruction, severe haemolytic anaemia, or thrombocytopaenia [2].

There are rare genetic predispositions to severe and atypical EBV infection. The most common, X-Linked Lymphoproliferative Disease (XLP), affects boys and is characterised by polyclonal $\mathrm{B}$ - and T-cell proliferation in response to EBV. Patients with XLP frequently develop fulminant hepatitis and haemophagocytic syndrome. Other pathological conditions linked with EBV include haemophagocytic lymphohistiocytosis (discussed separately), oral hairy leucoplakia, an increased risk of Hodgkin's lymphoma [3], nasopharyngeal carcinoma, Burkitt's lymphoma [4], and multiple sclerosis [5].

\section{Case report}

A 3.5-year-old girl was admitted to hospital with a suspicion of lymphoproliferative disorder. In the emergency room the child was ill-looking and fevered to $40^{\circ} \mathrm{C}$. Physical examination revealed cervical and submandibular lymphadenopathy, enlarged pharyngeal tonsils, and hepatosplenomegaly that was confirmed by ultrasonography. Blood test showed high leucocytosis $(61,600$ cells $/ \mu 1)$, mildly elevated uric acid level $(6.9 \mathrm{mg} / \mathrm{dl})$, and high LDH (1708 UI/l) and ALT (67 UI/1). Peripheral blood smear showed massive lymphocytosis with a $62 \%$ share of atypical lymphocytes. Bone marrow cytology corresponded to reactive hyperplasia. On the second day IgM antibodies to $\mathrm{CMV}$ and $\mathrm{EBV}$ VCA were positive suggesting double infection.

Polymerase chain reaction (PCR) examination revealed a massive replication of EBV (10700 copies/ml in plasma) and an unexpected lack of CMV DNA, which made our initial diagnosis questionable. After the primary decrease, leucocytosis reached $121,700 / \mu \mathrm{l}$ (83\% of atypical lymphocytes) in the fourth day of hospitalisation. Immunophenotyping of blood lymphocytes revealed 73,264 CD8+ CD3+ cells/ $\mu \mathrm{l}, 5581 \mathrm{NK}$ cells/ $\mu 1,10,854 \mathrm{CD} 4+\mathrm{CD} 3+$ cells $/ \mu 1$, and the CD4+/CD8+ ratio was 0.14 . Trepanobiopsy and lumbar puncture was performed. There was no evidence of lymphoproliferative disease.
The final diagnosis was infectious mononucleosis. Supportive treatment was administered, and after three weeks the child was discharged and monitored in the outpatient clinic. In order to explain such fulminant course of the infection, a standard screening for immunodeficiency was performed. After two years, despite a lack of any concomitant infection, immunophenotyping showed mild and persistent activation of CD8+ and NK cells, still decreased CD4+/CD8+ ratio (0.8), and questionable share of perforin+ cells. Repeated virological tests confirmed the absence of CMV IgM/IgG and seroconversion of IgG EBNA.

\section{Discussion}

The natural history of IM states that the majority of clinical and laboratory findings resolve spontaneously within one month of acute onset. Serious complications like airway obstruction, meningoencephalitis, haemolytic anaemia, thrombocytopaenia, and splenic rupture occurs in $1 \%$ of patients. Adults have an increased risk of fulminant course, which has been linked to abundant CD8+ T-cells activation against infected B-cells and following tissue infiltration [6]. At the same time, because of EBV immortalising properties, lack of adequate antiviral immune response may result in dangerous lymphoproliferative disease [3]. Surprisingly, clonal expansion of CD8+ T-cell, not the level of viremia, is correlated with the severity of symptoms. Cross-reaction between EBV-specific T-cells and host antigens can cause lysis of uninfected cells and lead to disease aggravation [7].

It is still not clear what exactly triggers T-cell clonal proliferation. Animal model studies on heterologous immunity between viruses revealed that pre-existing memory T-cells may contribute to excessive immune response during subsequent infection with new, unrelated pathogens. Then the risk of severe IM may be related to increasing memory CD8+ T-cell population with age [6]. The prevalence of multiple or subsequent infections with other respiratory agents is estimated at more than $60 \%$ of children with primary EBV infection [8]. It has been shown that cross-reactive CD8+ T-cells recognise epitopes of influenza M1 and EBV BMLF1. In effect, Influenza A-specific CD8+ T-cells participate in EBV-induced lymphoproliferation [6].

According to epidemiological data [5], primary infection with EBV and CMV usually occurs during preadolescence. Both viruses can infect immunocompetent individuals simultaneously, causing similar clinical presentation. Previous studies have reported that dual infection can elicit massive response of CD8+ T-cells, immunosuppression, hypercytokinaemia, and increased risk of severe complications [9]. Nevertheless, the CMV IgM result turned out to be false-positive. It has been proven in in vitro studies that CMV proteins used as recombinant ELISA antigens may react with IgM antibodies raised against other herpes- 
viruses. Epstein-Barr nuclear antigen 1 (EBNA-1) contains repetitive Gly-Ala elements, which are a major antigenic determinant for $\operatorname{IgM}$ antibody production during the acute phase of infection. The comparison of EBV and CMV antigen structure displayed homology between glycine-rich motifs of the CMV polypeptides (UL 112-113 family) and Gly-Ala repeat within the N-terminal half of EBNA-1. This may lead to misinterpretation [10].

Over time EBV has developed strategies to enhance the host's immune response. Homological to IL-10, the EBV BCRF1 protein is able to mimic IL-10-mediated inhibition of interferon- $a$ (INF- $a$ ), which successfully diminishes virus clearance [4]. Strong inflammatory response has been linked to polymorphisms of IL-1 and IL-10 genes $[11,12]$.

Epstein-Barr virus-Associated Haemophagocytic Lymphohistiocytosis (EBV-HLH) is a rare, life-threatening syndrome with exponential lymphocyte and histocyte activation and subsequent invasion of key components of the lymphatic system. The main pathological feature of HLH is monoclonal proliferation of CD8+ T-cells, which has been linked to mutations in perforin (PRF1) and in proteins that control perforin mobilisation from cytotoxic cells $\left(\mathrm{MUNC}_{13-14}, \mathrm{UNC}_{13} \mathrm{D}\right)$. Perforin is a cytolytic protein expressed mostly in granules of CD8+ lymphocytes and NK cells. Although our patient did not fulfil the baseline HLH diagnostic criteria, an increased share of perforin+ CD8+ T-cells and decreased perforin + CD56+ NK cells corresponds with findings in asymptomatic family members of children with HLH. The significance of such a phenomenon remains undetermined $[4,13]$.

Our case reflects the diagnostic difficulties in infectious mononucleosis. The network of reciprocal dependencies between viruses and the host's immune condition seems to be still an unexplored area.

The authors declare no conflict of interest.

\section{References}

1. Balfour HH, Dunmire SK, Hogquist KA (2015): Infectious mononucleosis. Clin Transl Immunol 4: e33.

2. Luzuriaga K, Sullivan L. Infectious mononucleosis (2010): N Engl J Med 362: 1993-2000.

3. Hjalgrim H, Askling J, Rostgaard K, et al. (2003): Characteristics of Hodgkin's Lymphoma after Infectious Mononucleosis. N Engl J Med 349: 1324-1332.

4. Bennett JE, Dolin R, Blaser MJ. Mandell, Douglas, and Bennett's Principles and Practice of Infectious Diseases. $8^{\text {th }}$ ed. Elsevier, Philadelphia 2015.

5. Handel AE, Williamson AJ, Disanto G, et al. (2010): An updated meta-analysis of risk of multiple sclerosis following infectious mononucleosis. PLoS One 5: pii: e12496.

6. Clute SC, Watkin LB, Cornberg M, et al. (2005): Cross-reactive influenza virus specific $\mathrm{CD} 8+\mathrm{T}$ cells contribute to lymphoproliferation in Epstein-Barr virus-associated infectious mononucleosis. J Clin Invest 115: 3602-3612.

7. Silins SL, Sherritt MA, Silleri JM, et al. (2001): Asymptomatic primary Epstein-Barr virus infection occurs in the absence of blood T-cell repertoire perturbations despite high levels of systemic viral load. Blood 98: 3739-3744.

8. Wang X, Yang K, Cong W, et al. (2010): Coinfection with EBV/CMV and other respiratory agents in children with suspected infectious mononucleosis. Virol J 7: 247.

9. Wakiguchi H, Hisakawa H, Kubota H, et al. (1999): Strong response of $\mathrm{T}$ cells in infants with dual infection by Epstein-Barr virus and cytomegalovirus. Pediatr Int 41: 484-489.

10. Lang D, Vornhagen R, Rothe M, et al. (2001): Cross-Reactivity of Epstein-Barr Virus-Specific Immunoglobulin M Antibodies with Cytomegalovirus Antigens Containing Glycine Homopolymers. Clin Diagn Lab Immunol 8: 747-756.

11. Hurme M, Helminen M (1998): Polymorphism of the IL-1 gene complex in Epstein-Barr virus seronegative and seropositive adult blood donors. Scand J Immunol 48: 219-222.

12. Helminen M, Lahdenpohja N, Hurme M (1999): Polymorphism of the interleukin-10 gene is associated with susceptibility to Epstein-Barr virus infection. J Infect Dis 180: 496499.

13. Kogawa K, Lee S, Villanueva J, et al. (2002): Perforin expression in cytotoxic lymphocytes from patients with hemophagocytic lymphohistiocytosis and their family members. Blood 99: 61-66. 